\title{
A Concept Design for an Ultra-Long-Range Survey Class AUV
}

\author{
Maaten E. Furlong \\ National Oceanography Centre, \\ Southampton \\ European Way, \\ Southampton. SO14 3ZH \\ Email: M.Furlong@noc.soton.ac.uk
}

\author{
Stephen D. McPhail \\ National Oceanography Centre, \\ Southampton \\ European Way, \\ Southampton. SO14 3ZH \\ Email: S.McPhail@noc.soton.ac.uk
}

\author{
Peter Stevenson \\ National Oceanography Centre, \\ Southampton \\ European Way, \\ Southampton. SO14 3ZH \\ Email: P.Stevenson@noc.soton.ac.uk
}

\begin{abstract}
Gliders and flight-style Autonomous Underwater Vehicles (AUVs) are used to perform perform autonomous surveys of large areas of open ocean. Glider missions are characterized by their profiling flight pattern, slow speed, long range $(1000 \mathrm{~s}$ of $\mathbf{~ k m})$ and many month mission duration. Flight-style AUV missions are faster, of shorter range (100s of $\mathrm{km}$ ) and multi day duration. An AUV combining many aspects of both vehicle classes would be of considerable value.

This paper investigates the factors that affect the range of a traditional flight-style AUVs. A generic range model is outlined which factors in the effects of buoyancy on the range. The model shows that to create a very long range AUV it is necessary to reduce the hotel load on the AUV to the order of $1 \mathrm{~W}$ and to add wings to overcome the vehicle's positive buoyancy whilst travelling at the reduced speed required for long range.

Using this model a concept long range AUV is outlined that is capable of travelling up to $5000 \mathrm{~km}$. The practical issues associated with achieving this range are also discussed.
\end{abstract}

\section{INTRODUCTION}

Autonomous large areas surveys of the ocean are currently carried out using either flight-style propeller-driven AUVs or undersea gliders. Both vehicle types, with the exception of the Slocum thermal glider [1], use chemical batteries as their energy store but differ in their propulsion method. Flightstyle AUVs use an electric motor and propeller to drive them through the oceans, tend to fly between $1.0-2.5 \mathrm{~m} / \mathrm{s}$, and have a range of up to $900 \mathrm{~km}$ (Autosub3) with a mission duration of approximately 8 days. On the other hand gliders use a buoyancy engine to propel themselves, typically travel between $0.2-0.45 \mathrm{~m} / \mathrm{s}$, have a range of up to $4800 \mathrm{~km}$ with a mission duration of up to 200 days (SeaGlider) [2]. Due to the nature of their propulsion system gliders are restricted to see-saw flight profiles, whereas flight-style AUVs are not.

It is tempting to think that gliders are inherently more efficient than flight-style AUVs, due to the substantial difference in range. However, this is not the case. Although both vehicle types use the same energy storage (batteries) gliders achieve their long range through their very slow speed (hence minimal propulsion power), and very low hotel load. The mean power used by the SeaGlider is of the order of $0.5 \mathrm{~W}$ [3] with propulsion power typically comprising $60 \%-85 \%$ of this value [4]. This compares with $45 \mathrm{~W}$ for the Remus 100 vehicle when travelling at its optimal cruise speed (calculated from the Remus100 specification) and $255 \mathrm{~W}$ for Autosub when travelling at $1.3 \mathrm{~m} / \mathrm{s}[5]$.

The substantially reduced power requirement for gliders compared to flight-class AUVs is the explanation for their increased range. The actual efficiency of the buoyancy engine for a glider is at best 50\% (occurring during the deepest diving profile) [4], which is comparable to that achieved by the electric motor and propeller propulsion system of flight-style AUVs.

A flight-class AUV, with such a low hotel power, and a much reduced cruise speed, would have a range performance comparable to that of a glider, but would have the advantages of a much larger speed range (allowing it to increase speed, for example, in areas of higher current), and it would not be restricted to the profiling flight patterns of a glider. Such a vehicle would combine many of the advantages of both vehicle classes.

The benefits of this class of ultra-long-range AUV inspired the Underwater Systems Lab of the National Oceanography Centre, Southampton to apply for funding to develop a vehicle of this class as part of the Oceans2025 research programme. This funding bid has been successful and a prototype vehicle is to deployed by 2012 . The vehicle will be designed to dive to $6000 \mathrm{~m}$ and have a range of $5000 \mathrm{~km}$.

An ultra-long-range AUV of this class will be ideally suited to carry out a very wide range of oceanographic missions. Its long range and sprint capability make practical its launch and recovery in sheltered coastal bays and make long reciprocal transects possible. Some examples of potential missions are: the Drake passage choke point; transects of ocean basins such as the 24 degree North Ellett line; and station keep at various locations, acting as a virtual mooring.

This paper discusses the work undertaken in the concept design of the ultra-long-range AUV. The paper highlights the key design challenges, and how they are proposed to be tackled.

Sections II-IV describes the range models of the AUV, while Section V outlines the concept AUV design and its modelled performance. Finally Section VI discusses the practical issues that need to be addressed in such a design. 


\section{Modelling AN AUV'S RANGE VS FORWARD SPEED}

To design an AUV which would meet the required performance specification it is necessary to understand the factors which affect the range of the vehicle. The range $(R)$ of an AUV can be calculated from the available battery energy $(E)$ and the power drawn at a given vehicle horizontal speed $(\dot{x})$. Here $\dot{x}$ is used instead of the more conventional $u$ to differentiate the horizontal speed $(\dot{x})$ from the speed along the axis of the vehicle $(u)$ which is affected by vehicle pitch. Once $E, \dot{x}$ and the battery power are known the range is simply:

$$
R=\frac{E}{P_{P}+P_{H}} \cdot \dot{x}
$$

Where, the battery power draw comprises two components the propulsion power $\left(P_{P}\right)$, which is defined as the power going directly into the propulsion motor and the hotel load $\left(P_{H}\right)$ defined as the power used by the onboard systems computers, sensors etc.

Determining the hotel load and available battery energy for a real vehicle is relatively straightforward, however determining the propulsion power is more of a challenge as it is a function of the vehicle's configuration and its motion through the water.

The hotel load, although not necessarily constant in time for a real AUV, is assumed to be constant within this model.

To simplify the problem of determining propulsion power $P_{P}$ we restricted the AUV motion to the vertical plane and assumed that the vehicle maintains constant depth. Hence turning and depth changes were ignored. Under these circumstances the propulsion power is modelled as a function of forward speed and is defined as:

$$
P_{P}=\frac{X_{N} \dot{x}}{\eta_{m t r} \eta_{\text {prop }}}
$$

where $X_{N}$ is the propeller thrust, $\dot{x}$ is the vehicle forward speed and $\eta_{m t r}$ and $\eta_{\text {prop }}$ are the efficiencies of the motor and propeller respectively.

\section{Simple AUV RANGE Model}

Assuming that the AUV is neutrally buoyant the drag on the vehicle, and hence required propeller thrust $X_{N}$, can be modelled as:

$$
X_{N}=\frac{1}{2} \rho \dot{x}^{2} \nabla^{\frac{2}{3}} C d_{\nabla}
$$

Where, $C d_{\nabla}$ is the volumetric drag coefficient, $\nabla$ is the vehicle's form volume, and $\rho$ is the density of the sea water. By combining equations (1), (2) and (3) the range of an AUV can be calculated for a given forward speed.

The maximum range for the AUV can be found by differentiating the range with respect to forward speed and equating to zero. However, if we differentiate the range with respect to the propulsion power and equate to zero, as in equation (4) we can determine the relationship between $P_{P}$ and $P_{H}$.

$$
\frac{d R}{d P_{P}}=0
$$

At the maximum range

$$
P_{P}=\frac{1}{2} P_{H}
$$

This relationship between power and hotel load allows us to easily determine the speed at maximum range, and hence calculate the maximum range for a given hotel load. As an example the maximum range of a neutrally buoyant Autosub with different hotel loads was calculated using the following coefficients.

$$
\begin{aligned}
C d_{\nabla} & =0.045 \\
\nabla & =3.43\left[\mathrm{M}^{3}\right] \\
\eta_{m t r} & =0.7 \\
\eta_{\text {prop }} & =0.7 \\
\rho & =1025 \\
\text { Energy } & =60[\mathrm{kWhrs}]
\end{aligned}
$$

The results of this hotel load vs range analysis are shown in Fig. 1.
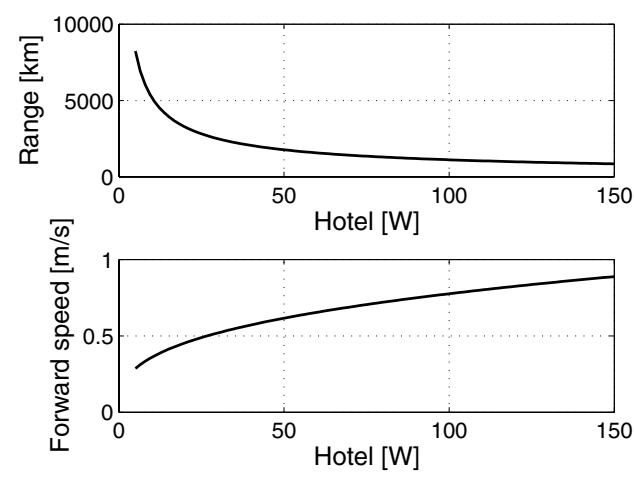

Fig. 1. Maximum range and the associated forward speed for Autosub vs hotel load using the simple range model

This simple range model shows that for Autosub to achieve the desired range of $5000 \mathrm{~km}$ it would be necessary to reduce the vehicle hotel power to approximately $10.5 \mathrm{~W}$ and the vehicle would have to travel at approximately $36.5 \mathrm{~cm} / \mathrm{s}$. This reduced hotel power has a significant impact on the sensors and instrumentation that can be used on an AUV. The implications of this are discussed later in the paper in reference to the practical issues associated with the long range AUV.

The simple range model predicts that a neutrally buoyant Autosub could achieve a range of $5000 \mathrm{~km}$, provided the hotel load was suitably reduced. However, Autosub and other flightstyle AUVs are not neutrally buoyant. This is because, in general, AUVs are designed to be positively buoyant to aid recovery and to cause the vehicle to float on the surface in the event of total system failure. Also, even if an AUV was designed to be neutrally buoyant, variation in sea water densities during the mission would causes changes in the vehicles buoyancy. Hence neutral buoyancy is not a reasonable assumption for AUVs. 
The buoyancy is important as it has a big impact on the flight performance of the AUV. Flight-style AUVs overcome the positive buoyancy by flying slightly nose down and using the lift generated by their body to counteract the buoyancy force. The lift of the body at constant pitch is proportional to the square of the forward velocity. As the vehicle slows the pitch has to increase to counteract the buoyancy. As the AUV slows further a limit is reached where the lift of the body cannot counteract the buoyancy and the vehicle can no longer maintain depth. Thus although the simple model predicts that a neutrally buoyant Autosub could travel 5000km it would be impractical for the real vehicle to fly at the slow speeds required. Hence, the maximum ranges predicted by the simple range model produces unrealistic results on buoyant AUVs.

For all its faults the simple model does however highlight the pivotal role played by the hotel load in achieving an extended AUV range.

To study the effect of buoyancy on range a more complex model was developed and is outlined in the next section.

\section{A Buoyancy-Adjusted Range Model}

When modelling the effect of buoyancy on range we needed to know how the hydrodynamic force and moments experienced by an AUV would vary with angle of attack. Standard submarine equations such as those given in [6], [7] model the hydrodynamic forces and moments experienced by an underwater vehicle in six degrees of freedom using experimentally derived hydrodynamic coefficients. These coefficients are laborious and expensive to determine, but have been determined for the Autosub Demonstrator Test Vehicle [8]. Thus, using the Autosub derived coefficients and submarine equations an estimate of the hydrodynamic forces and moments can be calculated for a vehicle geometrically similar to Autosub. As only steady state flight in the vertical plane at constant depth was of interest the submarine equations were greatly simplified.

The submarine equations use a body fixed and an inertial axis systems when modelling AUVs. These two axis systems are shown in Fig. 2 with $\dot{x}$ and $\dot{z}$ representing velocities in the inertial frame and $u$ and $w$ representing velocities in the body fixed reference frame.

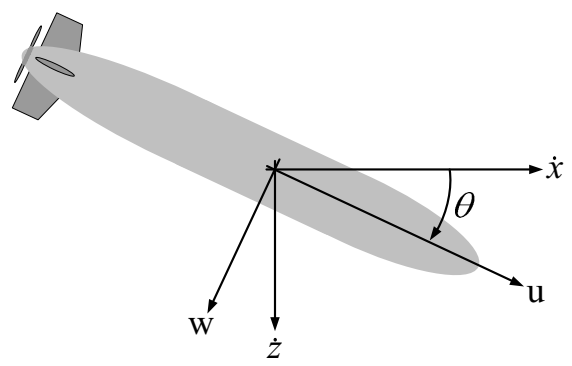

Fig. 2. Co-ordinate system used in modelling the AUV
Transformation between the two axis systems is performed using the rotation matrix shown in Equation (5) where $\theta$ is the pitch angle of the AUV.

$$
\left[\begin{array}{cc}
\cos (\theta) & -\sin (\theta) \\
\sin (\theta) & \cos (\theta)
\end{array}\right]\left[\begin{array}{c}
u \\
w
\end{array}\right]=\left[\begin{array}{c}
\dot{x} \\
\dot{z}
\end{array}\right]
$$

As a torpedo shaped body would not be capable of providing enough lift to overcome the positive buoyancy at low speeds it was necessary to add wings to the vehicle. The forces and moments generated by the wings could then be added to the forces and moments produced by a body using the approach outlined by [9]. Although the wings will affect the flow around the body and hence influence the force and moments it generates, it was assumed that this effect would be small and could be ignored without a significant loss of accuracy.

The forces and moments $X, Z$, and $M$ from the standard submarine equations and the forces and moment $D, L$ and $M_{w}$ produced by the added wings are shown in Fig. 3 .

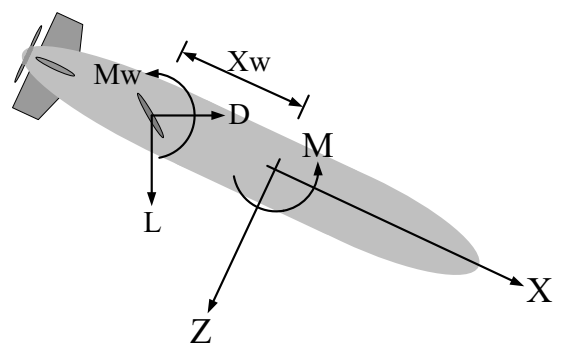

Fig. 3. Force and moments axis in the modelling

The submarine equations model the forces and moments in the body fixed coordinate system, while the wing forces and moments are modelled in the inertial frame.

The submarine equation forces and moment are modelled using the following equations:

$$
\begin{aligned}
X & =\frac{1}{2} \rho l^{2}\left(X_{u u}^{\prime} u^{2}+X_{w w}^{\prime} w^{2}+X_{u u \delta \delta S}^{\prime} u^{2} \delta S^{2}\right) \\
& +(B-M g) \sin \theta+X_{N} \\
Z & =\frac{1}{2} \rho l^{2}\left(Z_{u w}^{\prime} u w+Z_{w|w|}^{\prime} w|w|+Z_{u u \delta S}^{\prime} u^{2} \delta S\right) \\
& +(M g-B) \cos \theta \\
M & =\frac{1}{2} \rho l^{3}\left(M_{u w}^{\prime} u w+M_{w|w|}^{\prime} w|w|+M_{u u \delta S}^{\prime} u^{2} \delta S\right) \\
& -\left(M g x_{G}-B x_{B}\right) \cos \theta-\left(M g z_{G}-B z_{B}\right) \sin \theta
\end{aligned}
$$

where

- $X_{u u}^{\prime}, X_{w w}^{\prime}$, etc. are hydrodynamic coefficients of the AUV body.

- $B$ is the buoyancy force from the displaced water [N].

- $M g$ is the weight in Newtons of the vehicle [N]. 
- $l$ is the vehicle length $[\mathrm{m}]$.

- $x_{G}, z_{G} \& x_{B}, z_{B}$ are the $x$ and $z$ positions of the centres of gravity and buoyancy respectively in the body fixed axis system [m].

- $u, w$ are the body fixed velocities [m/s]

- $\theta$ is the vehicle pitch [Rad]

- $\delta S$ is the dive plane angle [Rad]

The forces and moment produced by the added wings ( $L$ $\left.D \& M_{w}\right)$ are calculated using the equations

$$
\begin{aligned}
L & =-\frac{1}{2} \rho \dot{x}^{2} A_{R} C_{L_{w}} \\
D & =-\frac{1}{2} \rho \dot{x}^{2} A_{R} C_{D_{w}} \\
M_{w} & =\frac{1}{2} \rho \dot{x}^{2} A_{R} C_{M_{w}}
\end{aligned}
$$

The lift, drag and moment coefficients $\left(C_{L_{w}}, C_{D_{w}}, C_{M_{w}}\right)$ are calculated from the geometry of the wings using a series of empirical equations described in [10]. These equations were slightly modified as the $C_{L_{w}}$ and $C_{M_{w}}$ terms were asymmetric in that they would produce different value for positive and negative angles of attack. This followed from the cross flow drag effect on $C_{L_{w}}$ and $C_{M_{w}}$. The modification made is illustrated for the $C_{L_{w}}$ coefficient. The original equation is given in (6) and the modified version is given in (7).

$$
\begin{aligned}
C_{L_{w}} & =\left(\frac{\partial C_{L_{w}}}{\partial \alpha}\right) \alpha+\frac{C_{D_{c}}}{a}\left(\frac{\alpha}{57.3}\right)^{2} \\
C_{L_{w}} & =\left(\frac{\partial C_{L_{w}}}{\partial \alpha}\right) \alpha+\frac{C_{D_{c}}}{a}\left(\frac{\alpha|\alpha|}{57.3^{2}}\right)
\end{aligned}
$$

The equations are derived from the modelling of ship control surfaces and as such are suited for predicting performance of low aspect ratio wings. The equations take the wing span, chord at the root and tip, quarter chord slope and angle of attack for a given wing section and tip profile and produce the lift, drag and moment coefficients for the described control surface.

Using the submarine and wing equations the net forces and moment experienced by the AUV can be found for a given forward speed $(\dot{x})$, pitch angle $(\theta)$ and dive plane angle $(\delta S)$. Solving these equations under the constraints of straight and level constant depth flight the steady state $\operatorname{pitch}(\theta)$, dive plane angle $(\delta S)$ and motor thrust $\left(X_{N}\right)$ can be found. Where the straight and level constant depth constraints are:

$$
\begin{aligned}
\dot{z} & =0 \\
\dot{x} & =\text { constant } \\
X+L \cos \theta+D \sin \theta & =0 \\
Z-L \sin \theta+D \cos \theta & =0 \\
M+M_{w}-L \cdot x_{W} \cos \theta-D \cdot x_{W} \sin \theta & =0
\end{aligned}
$$

Having determined $X_{N}$ it is possible to combine this with a modified version of equation (2) where the propulsion power is defined as

$$
P_{P}=\frac{X_{N} u}{\eta_{m t r} \eta_{\text {prop }}}
$$

Combining this propulsion power with equation (1) the range of the AUV can be determined. This approach assumes that the propeller thrust and efficiency are not affected by the angle of the fluid inflow which results from the vehicle's pitch angle.

Another issue with the approach is that the equations do not take into account Reynolds number effects. This is potentially a problem as it is likely that the vehicle will be going through the laminar to turbulent transition region over its speed range.

\section{LONG-RANGe AUV CONCEPT Design}

The buoyancy-adjusted range model was used to investigate the range performance of various concept designs for the long range AUV. To facilitate this process and to reduce the solution space the following assumptions were made about the long range AUV:

1) The energy density of a neutrally buoyant rechargeable lithium polymer battery was $100 \mathrm{wHrs} / \mathrm{kg}$.

2) The hydrodynamic coefficients used in the model are those of the Autosub DTV, but the drag coefficient $X_{u u}^{\prime}$ was modified to be equivalent to $C d_{\nabla}=0.035$.

3) The vehicle geometry was a scaled version of the Autosub DTV.

4) The hotel load was $1 \mathrm{~W}$.

5) The vehicle was positively buoyant by $0.3 \%$ of its displaced volume.

6) The battery comprised $1 / 3$ of the vehicle dry mass.

7) The vehicle dry mass was $80 \%$ of the vehicle's displaced mass.

8) The vehicle was assumed to be unable to operate with $|\theta|>10^{\circ}$ or $|\delta S|>15^{\circ}$.

9) The wings were NACA0015 section and were square tipped.

These assumptions allowed the vehicle body to be defined solely by its length. While the wings were specified only by their span, root \& tip chord, angle of attack and position on the body. These values were adjusted to design a small vehicle that would meet the $5000 \mathrm{~km}$ range requirements. This design process resulted in the following model input parameters being identified as suitable for the long range AUV.

$$
\begin{aligned}
\text { Length }[\mathrm{m}] & =3 \\
\text { Wing span }[\mathrm{m}] & =2.27 \\
\text { Chord root }[\mathrm{m}] & =0.6 \\
\text { Chord tip }[\mathrm{m}] & =0.3 \\
\text { Angle of attack }[\mathrm{deg}] & =-3 \\
\text { Wing location } X w[\mathrm{~m}] & =0.171
\end{aligned}
$$


The resulting outline of the AUV is shown in Fig. 4.

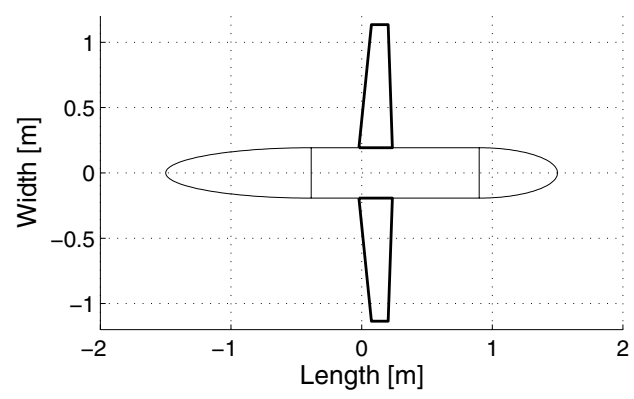

Fig. 4. Outline of the concept long range AUV

The identified model input parameters coupled with the model assumptions produce a vehicle with the following properties.

$$
\begin{aligned}
\text { Displaced volume }[\text { litres] } & =270 \\
\text { Battery Energy }[\mathrm{MJ}] & =26.6 \\
\text { Maximum range }[\mathrm{km}] & =5135 \\
\text { Endurance }[\text { days }] & =185
\end{aligned}
$$$$
\text { Speed at max range }[\mathrm{m} / \mathrm{s}]=0.32
$$

Dry mass $[\mathrm{kg}]=221$

The model also predicted the following range vs forward speed for this vehicle (Fig. 5) and the associated attitude vs range (Fig. 6).

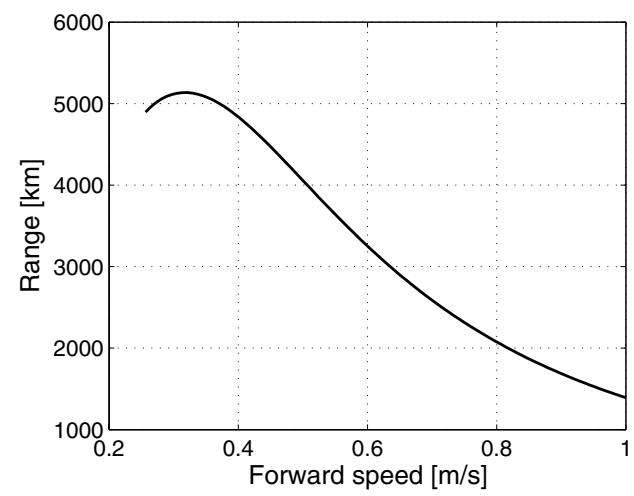

Fig. 5. Modelled range vs speed for the concept long range AUV

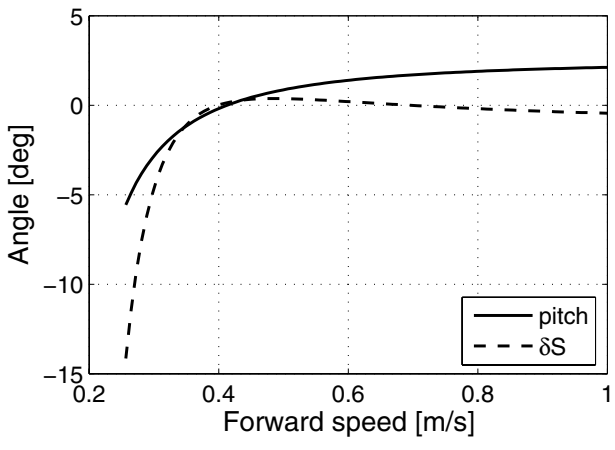

Fig. 6. Modelled attitude vs speed for the concept long range AUV

Studies were carried out to investigate the effect of changes in hotel load and changes in buoyancy on the maximum range of the AUV. The results of these studies are shown in Fig. 7 and Fig. 8 respectively.
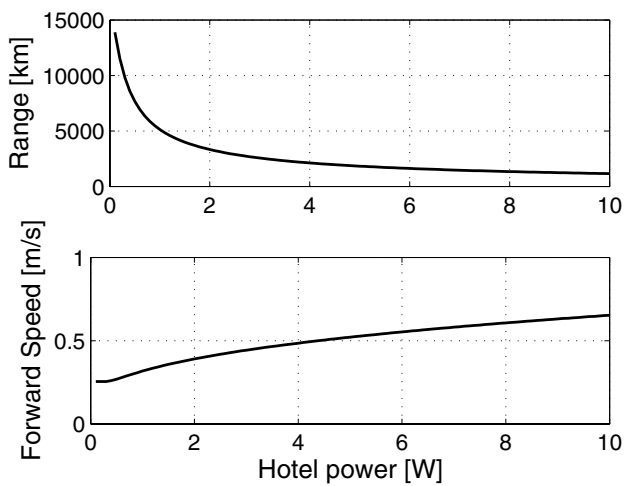

Fig. 7. Maximum range and associated forward speed for the long range concept AUV vs hotel load
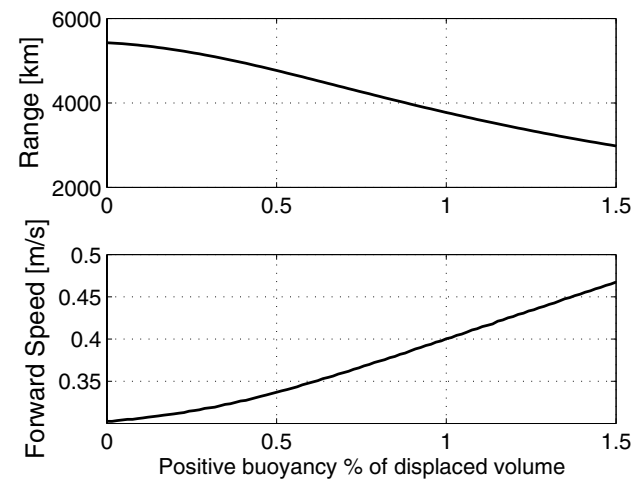

Fig. 8. Maximum range and associated forward speed for the long range concept AUV vs \% buoyancy

The sensitivity analysis shows that the range of the AUV is very highly affected by changes in the hotel load of the AUV, and significant range increases can be achieved by reducing the hotel load. The sensitivity analysis also shows how changes in the vehicle's net buoyancy will affect the maximum range of the AUV. Although the vehicle is less sensitive to buoyancy 
changes than to hotel load changes, an increase in the vehicle's net buoyancy will greatly reduce the total range of the AUV.

The results produced for the concept AUV, by the nature of the buoyancy-adjusted range model, are only an initial estimate of vehicle's range performance. The model, although compensating for buoyancy, still simplifies the modelling problem. The model does not take into account changes in the propeller performance arising from variations of water inflow into the propeller disc due to pitch changes. It also ignores Reynolds number effects which could be significant as the speed ranges involved fall within the laminar to turbulent transition region. Finally the model assumes that the propulsion motor maintains constant efficiency throughout its power range. These simplifications reduce the accuracy of the predictions, but the model does act as a good starting point for further more detailed design of the long range AUV.

\section{Practical Aspects of the Vehicle Design}

Having used the buoyancy-adjusted range model to produce a concept design for the long range AUV, it is necessary to examine some of the implications arising from the work. The first observation is the requirement to reduce the hotel load to $1 \mathrm{~W}$. This has a significant impact on the sensors that can be mounted on the vehicle, which in turn has an impact on vehicle navigation accuracy. Currently, highly accurate navigation can be achieved using a north seeking gyro to give accurate heading information and an acoustic doppler current profiler (ADCP) to give bottom track speed. This scheme is implemented on the Autosub AUV, using IXSEA's PHINS inertial navigation system (INS) to give the heading and RDI's Workhorse ADCP to give bottom track information. However, the PHINS INS requires $10 \mathrm{~W}$ of power, and hence couldn't be used on the long range AUV. To get an ADCP and INS which will work within the hotel load is a challenge, hence the navigation accuracy of the long range AUV is likely to be reduced compared to current flight-style AUVs. The reduced hotel load will also affect the sampling rate of the sensors on board the AUV. Currently CTDs sample at twenty times a second on Autosub but sample once every 5s on the Spray glider [11]. This reduced hotel power will require a navigation and sampling scheme more similar to that implemented on gliders than that used by current flight-style AUVs.

The second practical point to consider is handling, and launch \& recovery of the vehicle. As the concept vehicle is relatively large at $3 \mathrm{~m}$ and too heavy to manually lift at $221 \mathrm{~kg}$, the vehicle would need to be craned in and out of the water. Thus, the vehicle could only be operated from vessels with a suitable crane or would need to be shore launched. The large wings of the vehicle are also an issue as they are likely to be prone to damaged during launch and recovery operations. To mitigate the effects of this it would be necessary to make the wings weak points, so that in the event of an impact they break instead of the AUV.

\section{CONCLUSION}

A flight-style AUV that combined the range and endurance of a glider, while being able to fly at constant depth over a wide speed range would be a useful and flexible tool for the scientific community. As the buoyancy compensated range model has shown, it is feasible to produce such a vehicle. However, it is necessary for the vehicle to fly at $30-40 \mathrm{~cm} / \mathrm{s}$ to achieve these ranges, and wings would be required to overcome the positive buoyancy of the AUV. The hotel power of the AUV would also need to be reduced to the order of one Watt or so, and this has a direct impact on the sensors that can be fitted to the vehicle, and the control and navigation strategies that can be used.

The basic concept of a long range AUV capable of 5000km has been outlined, yet further analysis is required to fill in the details. It will be necessary to consider the effects of changes in efficiency of the propulsion system, both motor and propeller, at varying forward speeds and vehicle pitch angles. Further hydrodynamic analysis is also required to optimise the wing and body shape to maximise the range and controllability for a given vehicle size. A careful consideration needs to be given to the sensors packages, and usability of the vehicle.

Although much needs to be done in developing this concept a working prototype is planned to be produced by 2012 .

\section{REFERENCES}

[1] D. C. Webb, P. J. Simonetti, and C. P. Jones, "Slocum: an underwater glider propelled by environmental energy," Oceanic Engineering, IEEE Journal of, vol. 26, no. 4, pp. 447-452, 2001, 0364-9059.

[2] D. L. Rudnick, R. E. Davis, C. C. Eriksen, D. M. Fratantoni, and M. J. Perry, "Underwater gliders for ocean research," Marine Technology Society Journal, vol. 38, no. 2, 2004.

[3] C. C. Eriksen, T. J. Osse, R. D. Light, T. Wen, T. W. Lehman, P. L. Sabin, J. W. Ballard, and A. M. Chiodi, "Seaglider: a long-range autonomous underwater vehicle for oceanographic research," Oceanic Engineering, IEEE Journal of, vol. 26, no. 4, pp. 424-436, 2001, 0364-9059.

[4] G. Griffiths, Technology and applications of autonomous underwater vehicles, ser. Ocean science and technology, 1561-5928 ; v. 2. London: Taylor \& Francis, 2003, gBA310745 bnb 2750 edited by Gwyn Griffiths. ill. (some col.) ; $26 \mathrm{~cm}$. Includes bibliographical references and index.

[5] M. Furlong, "M408 mission summary," National Oceanography Centre, Southampton, Tech. Rep., 21st July 20062006.

[6] J. Feldman, "DTNSRDC revised standard submarine equations of motion," David W. Taylor Naval Ship Research And Development Center, Bethesda, MD, Tech. Rep. DTNSRDC/SPD-0393-09, 1979.

[7] A. Healey and D. Lienard, "Multivariable sliding mode control for autonomous diving and steering of unmanned underwater vehicles," Oceanic Engineering, IEEE Journal of, vol. 18, no. 3, pp. 327-339, 1993.

[8] N. Kimber and K. Scrimshaw, "Hydrodynamic testing of a $3 / 4$ scale autosub model," in Oceanology International 94, vol. 4. New Malden, UK.: Spearhead Exhibitions, 1994, p. unpaginated.

[9] M. Nahon, "A simplified dynamics model for autonomous underwater vehicles," in Autonomous Underwater Vehicle Technology, 1996. AUV '96., Proceedings of the 1996 Symposium on, 1996, pp. 373-379.

[10] E. V. Lewis, Principles of naval architecture, 2nd ed. Jersey City, N.J.: Society of Naval Architects and Marine Engineers, 1989, vol. III. Motion in Waves and Controllability.

[11] J. Sherman, R. E. Davis, W. B. Owens, and J. Valdes, "The autonomous underwater glider "spray"," Oceanic Engineering, IEEE Journal of, vol. 26, no. 4, pp. 437-446, 2001, 0364-9059. 